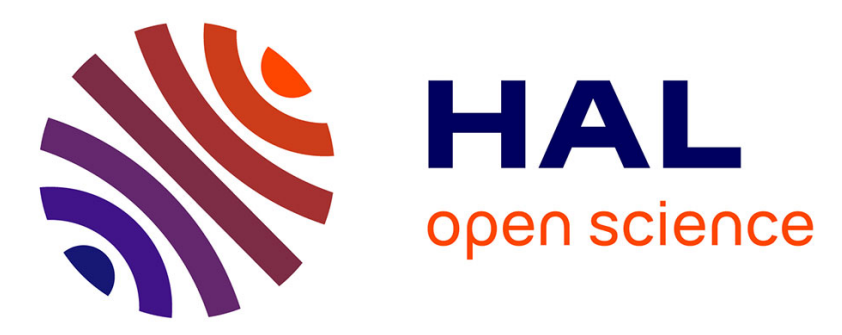

\title{
Medical Image Registration by Simulated Annealing and genetic algorithms
}

Samy Ait-Aoudia, Ramdane Mahiou

\section{To cite this version:}

Samy Ait-Aoudia, Ramdane Mahiou. Medical Image Registration by Simulated Annealing and genetic algorithms. Geometric Modeling and Imaging, 2007. GMAI '07, Jul 2007, Zurich, Switzerland. pp.145 - 148, 10.1109/GMAI.2007.25 . hal-00849156

\section{HAL Id: hal-00849156 https://hal.science/hal-00849156}

Submitted on $30 \mathrm{Jul} 2013$

HAL is a multi-disciplinary open access archive for the deposit and dissemination of scientific research documents, whether they are published or not. The documents may come from teaching and research institutions in France or abroad, or from public or private research centers.
L'archive ouverte pluridisciplinaire HAL, est destinée au dépôt et à la diffusion de documents scientifiques de niveau recherche, publiés ou non, émanant des établissements d'enseignement et de recherche français ou étrangers, des laboratoires publics ou privés. 


\title{
Medical Image Registration by Simulated Annealing and genetic algorithms
}

\author{
Samy Ait-Aoudia, Ramdane Mahiou \\ Institut National d'Informatique, BP 68M, Oued-Smar 16270 Algiers, Algeria \\ s_ait_aoudia@ini.dz,r_mahiou@ini.dz
}

\begin{abstract}
Registration techniques in medical image processing are used to match anatomic structures from two or more images (CT, MRI, PET,...) taken at different times to track for example the evolution of a disease. The core of the registration process is the maximization of a cost function expressing the similarity between these images. To resolve this problem, we have tested two global optimization techniques that are genetic algorithms and simulated annealing. In this paper we show some results obtained in medical images registration.
\end{abstract}

Keywords. Medical image registration, genetic algorithms, simulated annealing.

\section{Introduction}

Medical imaging is a vital part in disease diagnosis. Medical images provide information about pathology and related anatomy of the human body. Clinicians often wish to compare two or more images of the same anatomical regions acquired at different times to clinically track events. Medical image registration provides an important aid in diagnosis.

Image registration technique [13] can be defined as a mapping between two or more images spatially and with respect to similarity. For the sake of simplicity we admit that the registration will be made between an image to register $I_{R}$ and a reference image $I_{C}$.

Thus, the registration process consists in transforming the image to register $I_{R}$ such as the anatomic structures expressing the same information will be superposed in the two images $I_{R}$ and $I_{C}$ ([10], [5]). Formally, it is the maximization of the similarity (with respect to some criteria) between the two images [7].

In practice, the registration process comes to search the transformation $T$ that verifies the following relation:

$$
\text { Maximize[Similarity } \left.\left(I_{C}, T\left(I_{R}\right)\right)\right]
$$

In the preceding equation, the similarity (or similarity ratio) between an image $X$ and an image $Y$ is expressed by $\operatorname{Similarity}(X, Y)$ and $T(X)$ is the transformation of the $X$ image by $T$. To solve equation (1), we have tested two global optimization techniques that are genetic algorithms and simulated annealing.

This paper is organized as follows. We define in section 2 the principles of genetic algorithms. Simulated annealing is explained in explained in section 3 . Experimental results on medical samples datasets are given in section 4 . Section 5 gives conclusions.

\section{Genetic algorithms}

Genetic algorithms are the results of J. Holland works [3]. Holland tries to interpret and artificially model the principles of the Darwinian evolution.

\subsection{Description}

In an analogous manner to the Darwinian evolution, applying genetic algorithms to solve an optimization problem makes evolving a population of elements (individuals) (where every individual represent a solution) to a population optimizing this problem.

Evolutionary algorithms operate on a population of potential solutions applying the principle of survival of the fittest to produce better and better approximations to a solution. At each generation, a new set of approximations is created by the process of selecting individuals according to their level of fitness in the problem domain and breeding them together using operators borrowed from natural genetics. This process leads to the evolution of populations of individuals that are better suited to their environment than the individuals that they were created from, just as in natural adaptation.

For every individual, we can measure his adaptation ratio (fitness), which is the value of the 
function to optimize. For the evolving process, we use a "selection" operator, which chooses the best individuals of the actual population to put them in a "genetic basin". A "recombination" operator and a "mutation" operator are applied to the individuals in the "basin" to generate new individuals.

The general structure of an evolutionist genetic algorithm is the following [2]:

\begin{tabular}{|l|}
\hline Initialization; \\
Evaluation ; \\
Repeat \\
Evolution; \\
Selection; \\
Recombination; \\
Mutation ; \\
Evaluation; \\
Until (stop criterion). \\
\hline
\end{tabular}

The performance and complexity of a genetic algorithm narrowly depend on the following characteristics [9]:

- Coding: to define the intervention space of genetic operators (recombination and mutation).

- Population size.

- Fitness: the "quality" of a solution given by a cost function.

- Selection mode and reproduction operators.

- Other parameters (crossing over ratio, mutation ration, etc.)

\subsection{Coding}

In the following sections we represent an n-dimension solution by:

$$
X=\left\{x_{1}, x_{2}, \ldots, x_{n}\right\}
$$

- Natural coding: in this coding the characteristics of a solution are kept, an individual is represented by a vector of variables [11].

- Binary coding: consists in coding the characteristics of a solution by a string of bits [1]. It is a blind coding.

- Quantum coding: consists in replacing the bits of the binary coding by Qubits (quantum binary digit) [12]).

\subsection{Genetic operators}

\section{- Selection}

The aim of this step is to choose the best individuals for the reproduction. This choice can be deterministic or probabilistic. The selection is made independently of the coding scheme. The fitness of an individual is used to accomplish the task of this operator.

\section{- Recombination}

The recombination "crossover" operator allows the characteristics transmission from the parents to children.
The crossing-over is made with a given probability. The definition of this operator is narrowly linked to the coding used.

\section{- Mutation}

Mutation is a unary genetic operator that is characterized by the modification (with respect to a mutation probability) of the structure of an individual. Mutation operator is also linked to the coding used.

\section{- Stop criterion}

An optimization process based on a genetic algorithm converges if the actual population is homogeneous. A population is homogeneous if all or the majority of its individuals have the same genes.

\section{Simulated annealing}

Simulated annealing is a neighborhood based optimization method inspired from a technique used to have states of low energy of a material. This technique is called metallurgic or physics annealing.

\subsection{Description}

Inspired from the principles of the metallurgic annealing, Kirkpatrick [6] proposed an optimization method named simulated annealing. The energy of a material can be viewed as a cost function of the optimization problem. The different states of this material can be considered as solutions of the cost function.

The general structure of the simulated annealing algorithm is the following:

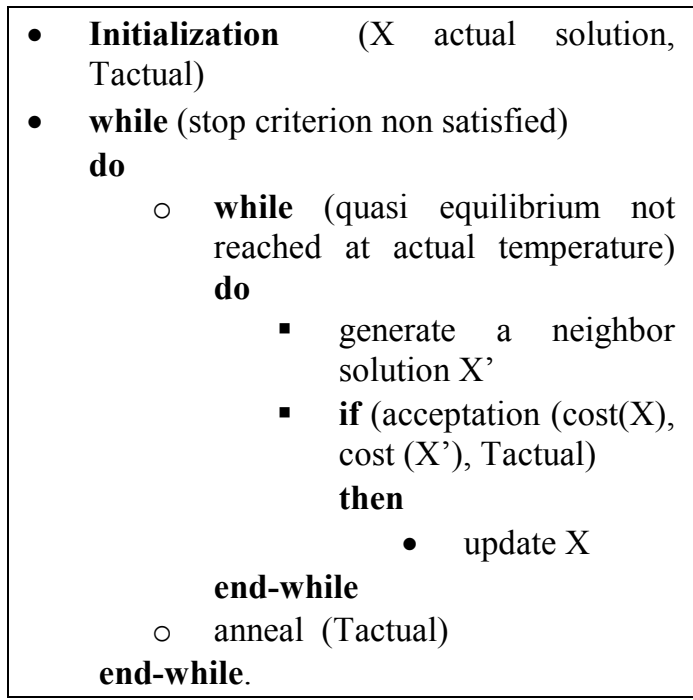

Initially the temperature is very high i.e. all the solutions of the search space are acceptable. The simulated processes by generating and accepting a neighbor solution until quasi equilibrium reached. Annealing will follow until satisfying a stop criterion.

Being probabilistic, the simulated annealing process can accept a solution that is worst than the actual solution. With this acceptation strategy, simulated annealing avoids the trap of local optima. Calculating an 
"admission" probability makes accepting a neighbor solution. The most used probability is metropolis criterion $[4,8]$. If $y$ is a neighbor solution to the actual solution $x$. The variation of energy given by $\Delta f=f(\mathrm{y})-$ $f(\mathrm{x})$ is calculated. The solution $y$ is accepted with respect to the probability (metropolis criterion) $p(x, y)$ given below:

$p(x, y)=e^{\left(\frac{f(y)-f(x)}{T}\right)}=e^{\left(\frac{-\Delta f}{T}\right)}$

\subsection{Simulated annealing characteristics}

\section{- Neighborhood}

The neighbor solution is calculated from a solution by applying some slight modifications. When the algorithm progresses, a visited solution can be considered again. Nevertheless, this probability is very low id the search space is large and the function generating neighbors is well conceived.

\section{- Temperature}

The temperature is a control parameter. When the temperature is high, new solutions can be admitted, while with low temperature global optimum is approached. The temperature must be decreased according to a function so a maximum of configurations can be tested.

\section{- Stop criterion}

During the progression of the optimization processes, the convergence toward the optimal solution cannot be determined. To overcome this difficulty, some criteria are used to appreciate the algorithm convergence. The most used criteria are:

- Rejected solutions ratio

- Cost variance of visited solutions

- Minimal temperature.

\section{Experimental Results}

All the tests were made on an IBM PC compatible machine (CPU Pentium 4, $1 \mathrm{GHz}$ and 256 Mo of RAM) under Microsoft Windows XP service pack2 operating system. The medical images used are acquired under different modalities. The following table shows some registration examples matching anatomic and functional images.
Table 1. Registration examples.

\begin{tabular}{|c|c|c|}
\hline Example & Reference Image & Image to register \\
\hline \#1 & & \\
\hline$\# 2$ & & \\
\hline \#3 & & \\
\hline
\end{tabular}

Table 2. Results of registration.

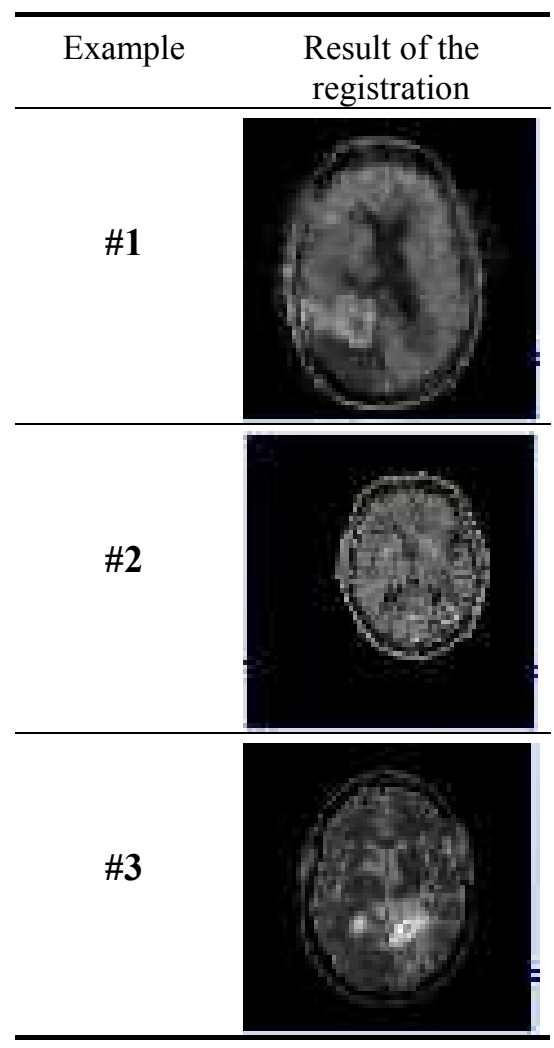




$$
C(I(.), J(T(.)))=-\sum_{g=1}^{G} \sum_{k=1}^{K} p(g, k) \cdot \log (g, k)
$$

The mutual entropy, given by equation (4) is a similarity measure largely used. $G$ and $K$ represent the number of gray tones of images $I$ and $J$. The following table gives the values of the mutual entropy for the three registration examples shown in table 1 . These results can be considered satisfying.

Table 3. Mutual entropy.

\begin{tabular}{cc}
\hline Example & Mutual entropy \\
\hline$\# 1$ & 4.04 \\
$\# 2$ & 2.23 \\
$\# 3$ & 3.12 \\
\hline
\end{tabular}

The registration tests were conducted using genetic algorithms (natural, binary and quantum coding) and simulated annealing (natural and binary coding) on the same samples. The following tables summarize the average costs of the registration process by using different optimization methods with different image sizes.

Table 4. Average registration time using genetic algorithms.

\begin{tabular}{|c|c|c|c|c|}
\cline { 3 - 5 } \multicolumn{2}{c|}{} & \multicolumn{3}{c|}{ Genetic algorithms } \\
\cline { 2 - 5 } \multicolumn{2}{c|}{} & $\begin{array}{c}\text { Natural } \\
\text { Coding }\end{array}$ & $\begin{array}{c}\text { Binary } \\
\text { Coding }\end{array}$ & $\begin{array}{c}\text { Quantum } \\
\text { Coding }\end{array}$ \\
\hline \multirow{3}{*}{$\begin{array}{c}\text { Image } \\
\text { size }\end{array}$} & $128 * 128$ & $31.21 \mathrm{~s}$ & $56.62 \mathrm{~s}$ & $57.31 \mathrm{~s}$ \\
\cline { 2 - 5 } & $256 * 256$ & $58.3 \mathrm{~s}$ & $73.05 \mathrm{~s}$ & $78.89 \mathrm{~s}$ \\
\cline { 2 - 5 } & $400 * 456$ & $70.81 \mathrm{~s}$ & $89.15 \mathrm{~s}$ & $96.61 \mathrm{~s}$ \\
\cline { 2 - 5 } & $600 * 700$ & $103.54 \mathrm{~s}$ & $125.12 \mathrm{~s}$ & $132.91 \mathrm{~s}$ \\
\hline
\end{tabular}

Table 5. Average registration time using simulated annealing.

\begin{tabular}{|c|c|c|c|}
\cline { 3 - 4 } \multicolumn{2}{c|}{} & \multicolumn{2}{c|}{ Simulated annealing } \\
\cline { 3 - 4 } \multicolumn{2}{c|}{} & Natural Coding & Binary Coding \\
\hline \multirow{3}{*}{$\begin{array}{c}\text { Image } \\
\text { size }\end{array}$} & $128 * 128$ & $6.52 \mathrm{~s}$ & $12.73 \mathrm{~s}$ \\
\cline { 2 - 4 } & $256 * 256$ & $11.63 \mathrm{~s}$ & $17.89 \mathrm{~s}$ \\
\cline { 2 - 4 } & $400 * 456$ & $24.03 \mathrm{~s}$ & $34.58 \mathrm{~s}$ \\
\cline { 2 - 4 } & $600 * 700$ & $46.5 \mathrm{~s}$ & $67.02 \mathrm{~s}$ \\
\hline
\end{tabular}

\section{Conclusion}

We have explored image registration in medical imaging. The registration process tests were conducted on the same medical image samples using genetic algorithms and simulated annealing. Simulated annealing with natural coding seems to be the fastest method. Further tests must be conducted on large samples and other optimization techniques must be tried to have a more accurate synthetic view of the registration process in medical imaging field.

\section{References}

[1] Andrey P., "Segmentation d'images par algorithmes génétiques", PhD Thesis, Paris 7 University, France, 1999.

[2] Harik G.R., Lobo F.G. \& Goldberg D.E., "The compact genetic algorithm", IEEE World Congress on Computational Intelligence, pp. 523-528, May 1998.

[3] Holland J.H.., "adaptation in natural and artificial systems", Michigan University Press, 1975.

[4] Johan D., "Métaheuristiques pour l'optimisation difficile", Eyrolles Edition, France, 2003.

[5] Juan D., Tang S., Jiang T. \& Lu Z., "Intensity-based robust similarity for multimodal image registration", International Journal of Computer Mathematics, vol. 83, $\mathrm{n}^{\circ}$ 1, Jan. 2006, pp. 49-57.

[6] Kirkpatrick S., Gelatt C.D. \& Vecchi M.P., "Optimisation by simulated annealing", Science, volume 220, numéro 4598, pp 671-680, 1983.

[7] Li W. \& Leung H., "A maximum likelihood approach for image registration using control point and intensity" IEEE Trans. on Image Processing, vol. 13, Issue 8, pp. 1115-1127, August 2004.

[8] Mendonca R.S. \& Caloba L.P., "New simulated annealing algorithms", proceedings ISCAS '97 vol.3, pp. 1668-1671.

[9] Miramond B., "Méthodes d'optimisation pour le partitionnement logiciel/matériel de systèmes à description multi-modèles", PhD Thesis, Evry University, France, Décembre 2003.

[10] Musse O., Heitz F. \& Armspatch J.P.., "Topology preserving deformable image matching using constrained hierarchical parametric models", International Conf. On Image Processing, vol. 1, pp.505-508, Sep. 2000.

[11] Salomon A., "Etude de la parallélisation de méthodes heuristiques d'optimisation combinatoire : application au recalage d'images médicales", $\mathrm{PhD}$ Thesis, Louis Pasteur University, Strasbourg, France, Décembre 2001.

[12] Talbi H., Dra A. \& Batouche M., "A new quantum inspired genetic algorithm for solving the salesman problem", IEEE international Conf. On Industrial Technology, 8-10 December 2004, vol. 3, pp. 1192-1197.

[13] Zitova B. \& Flusser J., "Image registration methods: a survey", Image and Vision Computing 21 (2003) 9771000 . 